\title{
THE REFLECTION OF SHORT WAVES IN A VARIABLE MEDIUM*
}

\author{
Bx J. J. MAHONY (University of Western Australia)
}

Approximate solutions for the one-dimensional propagation of waves in a slowly varying medium can be obtained quite simply by the W.K.B.J. method. Moreover careful error estimates have been obtained using the Langer related equation technique and these show that the W.K.B.J. solution is a valid asymptotic representation of the exact solution provided the wave speed is suitably regular and of bounded variation in $(-\infty, \infty)$. If the wave speed is a $C^{-}$function of position then it has been established that the error in the extended W.K.B.J. solution is smaller than any power of the parameter $\epsilon$, the ratio of wave length to the typical length scale associated with variations of the wave speed, for sufficiently small values of $\epsilon$. If however the wave speed is a piecewise $C^{m}$ function of position then the extended W.K.B.J. solution is valid with an error $O\left(\epsilon^{n+1}\right)$ so that for practical approximation purposes the W.K.B.J. solution would appear to be entirely satisfactory.

However there is one feature of the approximate solution which seems quite anomalous in a physical context and that is the result that for a $C^{-}$variation of wave speed with position an incident wave is modified in amplitude, wavelength and phase but no reflected wave is indicated even when there are significant total changes in wave speed. The only case when a reflected wave can be located by the W.K.B.J. method is when the wave speed is only piecewise $C^{\infty}$ but there are discontinuities in wave speed or its derivatives. In such cases the amount of reflected wave generated is governed by the order of the derivative where the discontinuity occurs, no matter how small the all over change associated with this discontinuity, which intuitively does not seem reasonable as a description of a physical phenomenon. However it must be remembered that the W.K.B.J. theory is valid in limit of vanishingly small $\epsilon$ and so sudden changes in wave shape can not be considered in this case. Particularly in the case of the reflection coefficient, $r$, when the W.K.B.J. solution gives the value 0 for $C^{\bullet}$ wave speed variations, but also in other cases it is desirable to have an estimate of the exponentially small error of the asymptotic series representation. Such estimates would give better indications as to what is the practical limit on $\epsilon$ in order to obtain answers of a given accuracy. Thus the present work is aimed at obtaining a suitable representation for the reflection coefficient for an arbitrary $C^{\infty}$ or analytic wave speed.

The mathematical formulation of this problem involves the solution of the ordinary differential equation

$$
\epsilon^{2}\left(d^{2} y / d x^{2}\right)+g(x) y=0, \quad-\infty<x<\infty,
$$

where $g$ is a strictly positive $C^{\infty}$ function of unit order while changes occur over unit scale in $x$. It is required to determine complex constants $r$ and $t$ such that for $x$ large negative

$$
y \sim \exp \left\{i[g(-\infty)]^{1 / 2} x / \epsilon\right\}+r \exp -\left\{i[g(-\infty)]^{1 / 2} x / \epsilon\right\}
$$

${ }^{*}$ Received October 6, 1966. This work was supported in part by the National Science Foundation under Grant GP5792, the Office of Naval Research under Contract Nonr 1866(34), and the division of Engineering and Applied Physics, Harvard University. 
and for $x$ large positive

$$
y \sim t \exp \left\{i[g(\infty)]^{1 / 2} x / \epsilon\right\}
$$

Actually because of coordinate uncertainties at $\infty$, the phases are not uniquely determined so it is really the moduli of the constants $r$ and $t$ which are of interest. It is convenient to use the Langer related equation technique to transform equation (1) into

$$
d^{2} Y / d \xi^{2}+Y=-\epsilon^{2} g^{-3 / 4}\left(d^{2} / d x^{2}\right)\left\{g^{-1 / 4}\right\} Y
$$

where

$$
\begin{gathered}
\xi=u(x) / \epsilon, \\
u(x)=\int_{0}^{x}\{g(t)\}^{1 / 2} d t
\end{gathered}
$$

and

$$
y=\{g(-\infty) / g(x)\}^{1 / 4} Y .
$$

The strictly positive $C^{\infty}$ nature of $g(x)$ makes, with the positive square root chosen, the relationship of $x$ in $(-\infty, \infty)$ and $\xi$ in $(-\infty, \infty)$ one-one and the regularity properties as functions of $\xi$ are the same as those as functions of $x$. If $Z(\xi)$ denotes

$$
Z(\xi)=\epsilon^{2}\left(d^{2} / d x^{2}\right)\{g(x)\}^{-1 / 4}\{g(x)\}^{-8 / 4} Y(\xi)
$$

then the formal solution of equation (4), subject to the incident wave condition of equation (2),

$$
Y(\xi)=e^{i \xi}+\frac{1}{2} i e^{i \xi} \int_{-\infty}^{\xi} e^{-i \xi^{\prime}} Z\left(\xi^{\prime}\right) d \xi^{\prime}+\frac{1}{2} i e^{-i \xi} \int_{\xi}^{\infty} e^{i \xi^{\prime}} Z\left(\xi^{\prime}\right) d \xi^{\prime}
$$

is obtained by variation of parameters. The integrals will converge for reasonable assumptions on the behavior of $g(x)$ near $\pm \infty$. The reflection and transmission coefficients are thus given by

$$
r=(i / 2) \int_{-\infty}^{\infty} e^{i \xi} Z(\xi) d \xi
$$

and

$$
t=\{g(-\infty) / g(\infty)\}^{1 / 4}\left\{1+(i / 2) \int_{-\infty}^{\infty} e^{-i \xi} Z(\xi) d \xi\right\}
$$

Reverting now to the use of $x$ to specify position we obtain for the reflection coefficient, where the factor $i$ can be ignored,

$$
r=(\epsilon / 2) \int_{-\infty}^{\infty} e^{i u(x) / 4}\{g(x)\}^{-1 / 4} \frac{d^{2}}{d x^{2}}\{g(x)\}^{-1 / 4} Y d x .
$$

Although not normally obtained in this way, the W.K.B.J. approximation is the series solution obtained by iteration of the integral Eq. (9) and expanding integrals asymptotically which is the way that error estimates have been established. This approximation takes the form

$$
Y \sim e^{i \xi}\left\{1+\sum_{n=1}^{\infty} \epsilon^{n} U_{n}(x)\right\}
$$


It is plausible to argue that any reflected wave which is produced, of smaller order than any power of $\epsilon$, will propagate in a similar fashion to the incident wave. If this is so then the wave traveling in the negative $x$-direction will be the simple resultant of all reflected waves produced at greater values of $x$ so that the reflected wave at any station should be of no greater order than $r$ and a more complete description of $Y$ should be

$$
Y \sim \exp (i u(x) / \epsilon) U(x, \epsilon)+r \exp (-i u(x) / \epsilon) R(x, \epsilon)
$$

where $R$ is of unit order at most. Substitution of this assumed behavior into Eq. (10) gives the result

$$
r \sim \frac{1}{2} \epsilon \int_{-\infty}^{\infty} \exp (2 i u(x) / \epsilon)\{g(x)\}^{-1 / 4} \frac{d^{2}}{d x^{2}}\{g(x)\}^{-1 / 4} d x\{1+0(\epsilon)\}
$$

where it has been assumed that the integral involving $R$ converges because of the steady approach of $g(x)$ as its limiting values at infinity. Equation (11) permits the consistent interpretation of the generation of reflected wave at each point by the dominant incident wave. Whereas it is particularly difficult to obtain $R(x, \epsilon)$ from Eq. (9), due to the fact that the last integral is dominated by the contribution from the lower terminal which yields a wave traveling in the same direction as the incident wave, Eq. (11) permits investigation by complex variable methods and for suitable functions $g(x)$ explicit dependance to leading order may be established. Thus if $\{g(x)\}$ is a rational algebraic function then $r$ will be $O\left(\epsilon e^{-\alpha / \epsilon}\right)$ where $\alpha$ is related to the imaginary part of the pole closest to the real axis. The value of $\alpha$, if it happens to be moderately small, will place a real restriction on the range of values of $\epsilon$ for which the W.K.B.J. solution is likely to be valid.

It is of interest to speculate whether even more restrictive types of transcendentally small behavior in $\epsilon$ can occur and the following rather trumped up variation, and rather heuristic asymptotic estimates, suggest that there is a genuine possibility of this being the case. Consider

$$
\begin{aligned}
g(x) & =1, & & x<0, \\
& =\left(1+\lambda \exp \left(-1 / x^{\alpha}\right)\right)^{-4}, & & x>0
\end{aligned}
$$

which will lead to integrals in the expression for the reflection coefficient of the form

$$
\int_{0}^{\infty} \exp \left\{-\frac{1}{x^{\alpha}}-\beta \ln x+i x / \epsilon+(i \lambda / \epsilon) \int_{0}^{x} \exp \left(-t^{-\alpha}\right) d t\right\} d x .
$$

Since the real part of the expression in the interior of the \{\} tends to $-\infty$ at both and points this integral suggests the application of the method of steepest descent. Moreover for $\alpha \leq 1$ the real part is always negative on the real axis so that at the appropriate col it must certainly be negative. It would seem that the appropriate col must be near the origin when $\ln x$ and the integral are unimportant so that the col would satisfy approximately

$$
x=(\alpha i \epsilon)^{1+\alpha}
$$

with the appropriate choice of branch. This would lead to a reflection coefficient whose transcendental part was like exp - $\left\{\delta(\alpha \epsilon)^{\alpha /(1+\alpha)}\right\}$ and it is apparent that for quite moderate values of $\alpha$, for example $\frac{1}{4}$, this term could be numerically significant, in com- 
parison with powers of $\epsilon$, even for $\epsilon$ of order (1/100). Therefore it would appear worthwhile when using W.K.B.J. method to make a quick estimate of the reflection coefficient in order to assess the likely restrictions on $\epsilon$ for the validity of the W.K.B.J. approximation. The possibility of numerical importance of transcendentally small terms almost certainly explains why the W.K.B.J. predicts reflection coefficients dependent of such physically unlikely quantities as the nature of the singularities of $g(x)$. 